\title{
Squeezing in Coupled Oscillators Having Neither Nonlinear Terms Nor Time-Dependent Parameters
}

\author{
H. Rodrigues, D. Portes Jr., \\ Centro Federal de Educação Tecnológica-CEFET/RJ, \\ CEP 20.271-110, Rio de Janeiro (RJ), Brazil \\ S.B. Duarte, \\ Centro Brasileiro de Pesquisas Físicas /CNPq, \\ CEP 22.290-180, Rio de Janeiro (RJ), Brazil \\ and B. Baseia \\ Instituto de Física, Universidade Federal de Goiás, \\ CEP 74.001-970, Goiania (GO), Brazil
}

Received on 14 February, 2001

\begin{abstract}
The occurrence of squeezing effects in coupled oscillators, and the transference between them, has been studied in various situations using Hamiltonians involving either nonlinear terms or timedependent parameters. We consider a simplified scheme generating this effect, and discuss its origin.
\end{abstract}

\section{Introduction}

Extensive study of squeezing has been developed since the last decade, both theoretically [1] and experimentally [2]. States of oscillators and single-mode of electromagnetic fields exhibiting this effect were named: squeezed states (Hollenhost [1]); two-photon coherent states (Yuen [1]); generalized coherent states (Stoler [1]); sub-fluctuant states (Glauber and Levenstein [3]); etc. They are nonclassical states, having reduced dispersion in one field-quadrature in comparison with that found in coherent states or vacuum state. Investigations of squeezing have been implemented in various contexts, e.g., considering the system composed by two interacting oscillators, where: (i) one of them has a time-dependent parameter, hence the squeezing effect is generated in such oscillator, its transference to the other oscillator being considered $[4,5,6]$; (ii) one of the oscillators is nonlinear, e.g., quadratic in the annihilation operator $\mathbf{a}$ and the creation operator $\mathbf{a}^{+}$, hence squeezing is created in this oscillator [1] its transference to the second (linear and t-independent) oscillator being studied, including optimization of this transference [7]; (iii) both oscillators are quadratic in $\mathbf{a}$ and $\mathbf{a}^{+}$, this being an extension of the approach presented in [7], as shown in Ref.[8]; (iv) both oscillators are linear and have no t-dependent parameter, but the coupling between them is t-dependent [9]. In all these cases the effect may refer either to 1-mode squeezing [1] or 2mode squeezing [10], the first scenario concerning with individual variables (e.g., the in a variance of a single oscillator); and the second scenario with collective variables (e.g., the effect in either the CM-coordinate or in the RELATIVE-coordinate[10]).

To our knowledge, no mention in the literature has been done about the occurrence of one-mode squeezing in coupled linear oscillators, having no t-dependence, the scenario seeming to be confined just to the case of two- mode squeezing. In this paper we will show that this is not always true, the result depending on the type of the interaction between the oscillators.

This report is organized as follows: In the Sect. II we present a brief summary of useful definitions and Hamiltonian-schemes employed previously in the literature. Sect. III is about the present model and calculations yielding our results. Sect. IV contains the comments and conclusion.

\section{Hamiltonian-models for cou- pled oscillators}

In Ref.[6] the following model was employed to investigate the generation and transference of squeezing from 
one oscillator to the other :

$$
\mathbf{H}=\mathbf{H}_{Q}\left(\mathbf{a}, \mathbf{a}^{+}\right)+\mathbf{H}_{L}\left(\mathbf{b}, \mathbf{b}^{+}\right)+\mathbf{V},
$$

where $\mathbf{H}_{Q}$ is a quadratic Hamiltonian describing the first oscillator

$$
\mathbf{H}_{Q}=\hbar \omega\left(\mathbf{a}^{+} \mathbf{a}+\frac{1}{2}\right)+\hbar f\left(\mathbf{a}^{+2}+\mathbf{a}^{2}\right),
$$

$\mathbf{H}_{L}$ is a linear Hamiltonian describing the second oscillator,

$$
\mathbf{H}_{L}=\hbar \omega\left(\mathbf{b}^{+} \mathbf{b}+\frac{1}{2}\right),
$$

and $\mathbf{V}$ stands for their interaction, assumed in Ref.[6] as usually

$$
\mathbf{V}=\hbar \lambda\left(\mathbf{a} \mathbf{b}^{+}+\mathbf{a}^{+} \mathbf{b}\right),
$$

where $\lambda$ is the coupling constant.

This form of interaction corresponds to what is called Rotating-Wave-Approximation (RWA), encountered in studies involving atom-field interaction. In this case the complete atom-field interaction in the Jaynes-Cummings model [11] reads [12]

$$
\begin{aligned}
\mathbf{V}^{\prime} & =\hbar \lambda \mathbf{p} . \mathbf{E}=\hbar \lambda\left(\mathbf{a}+\mathbf{a}^{+}\right)\left(\sigma^{+}+\sigma^{-}\right) \\
& =\hbar \lambda\left(\mathbf{a} \sigma^{+}+\mathbf{a}^{+} \sigma^{-}\right)+\hbar \lambda\left(\mathbf{a} \sigma^{-}+\mathbf{a}^{+} \sigma^{+}\right),
\end{aligned}
$$

where $\mathbf{p} \sim\left(\sigma^{+}+\sigma^{-}\right)$is the atomic dipole operator and $\mathbf{E} \sim\left(\mathbf{a}^{+}+\mathbf{a}\right)$ is the electric field operator.

Neglecting the counter-rotating term $\hbar \lambda\left(\mathbf{a} \sigma^{-}+\right.$ $\mathbf{a}^{+} \sigma^{+}$) leads to a good approximation when the condition $\lambda \sqrt{\bar{n}}<<1$ is satisfied [13], with $\bar{n}$ being the mean excitation number. In terms of quadrature operators $\left\{\mathbf{x}_{1}, \mathbf{p}_{1}\right\},\left\{\mathbf{x}_{2}, \mathbf{p}_{2}\right\}$, given by

$$
\begin{aligned}
& \mathbf{x}_{1}=\left(\mathbf{a}+\mathbf{a}^{+}\right) / 2, \quad \mathbf{p}_{1}=\left(\mathbf{a}-\mathbf{a}^{+}\right) / 2 i \\
& \mathbf{x}_{2}=\left(\mathbf{b}+\mathbf{b}^{+}\right) / 2, \quad \mathbf{p}_{2}=\left(\mathbf{b}-\mathbf{b}^{+}\right) 2 i
\end{aligned}
$$

the interaction in Eq.(4) results in the form

$$
\mathbf{V}=\hbar \lambda\left(\mathbf{x}_{1} \mathbf{x}_{2}+\mathbf{p}_{1} \mathbf{p}_{2}\right) .
$$

In Refs. $[7,8]$ it was shown that the squeezing effect generated in the quadratic oscillator can be transferred to the linear oscillator. For interesting details, as the mathematical procedure and optimization of such squeezing transfer, the reader is referred to $[7,8]$.

On the other hand, in Ref.[9] a different scheme was employed: both oscillators are linear, the squeezing effect emerging from the t-dependence of the coupling between them. In this case the Hamiltonian was modelled as follows [9]:

$$
\mathbf{H}=\frac{1}{2} \hbar \sum_{i}\left(\mathbf{p}_{i}^{2}+\mathbf{x}_{i}^{2}\right)+\hbar \lambda(t) \mathbf{x}_{1} \mathbf{x}_{2} .
$$

Note that the interaction in Eq.(9) can be written in the form

$$
\mathbf{V}=\hbar \lambda(t)\left(\mathbf{a b}^{+}+\mathbf{a}^{+} \mathbf{b}\right)+\hbar \lambda(t)\left(\mathbf{a} \mathbf{b}+\mathbf{a}^{+} \mathbf{b}^{+}\right),
$$

where the Eqs. $(6,7)$ have been used. For comparison, note also that an alternative interaction to that used in Eq.(9) is

$$
\mathbf{V}=\hbar \lambda(t) \mathbf{p}_{1} \mathbf{p}_{2} .
$$

In this case, application of Eqs.( 6, 7 ) gives

$$
\mathbf{V}=-\hbar \lambda(t)\left(\mathbf{a} \mathbf{b}^{+}+\mathbf{a}^{+} \mathbf{b}\right)+\hbar \lambda(t)\left(\mathbf{a} \mathbf{b}+\mathbf{a}^{+} \mathbf{b}^{+}\right) .
$$

A comparison of Eq.(10) and Eq.(12) shows a difference in the signals accompanying their rotating terms $\left(\mathbf{a b}^{+}+\mathbf{a}^{+} \mathbf{b}\right)$. On the other hand, the interaction used in Refs. $[7,8]$ did not include the counter-rotating term $\left(\mathbf{a} \mathbf{b}+\mathbf{a}^{+} \mathbf{b}^{+}\right)$. So, in this case the difference is more evident. It is interesting to mention that the rotatingwave term in Eqs.(10,12), written in terms of $\mathbf{x}_{i}, \mathbf{p}_{i}$, results

$$
\mathbf{a b}^{+}+\mathbf{a}^{+} \mathbf{b}=2\left(\mathbf{x}_{1} \mathbf{x}_{2}+\mathbf{p}_{1} \mathbf{p}_{2}\right),
$$

whereas the counter-rotating part in these equations reads, in terms of $\mathbf{x}_{i}$ and $\mathbf{p}_{i}$,

$$
\mathbf{a} \mathbf{b}+\mathbf{a}^{+} \mathbf{b}^{+}=2\left(\mathbf{x}_{1} \mathbf{x}_{2}-\mathbf{p}_{1} \mathbf{p}_{2}\right) .
$$

Note that Eqs.(13, 14 ) are consistent with the Eqs. $(9,10)$ and Eqs.(11, 12).

Distinct kinds of interactions lead to different results, e.g., variances exhibiting one-mode squeezing, or not. Here we will employ a convenient form in these alternatives to verify the occurrence of squeezing in our system.

\section{The interaction $\mathbf{V} \sim \mathbf{p}_{1} \mathbf{p}_{2}$}

In this section we shall investigate the occurrence of squeezing in the model-Hamiltonian

$$
\mathbf{H}=\mathbf{H}_{1}+\mathbf{H}_{2}+\mathbf{V},
$$

where $\mathbf{H}_{1}$ and $\mathbf{H}_{2}$ are independent oscillator Hamiltonians

$$
\mathbf{H}_{i}=\frac{\mathbf{p}_{i}^{2}}{2 m_{i}}+m_{i} \omega_{i}^{2} \mathbf{x}_{i}^{2} \quad, \quad i=1,2
$$

and $\mathbf{V}$ is the time-independent interaction

$$
\mathbf{V}=\frac{k}{\sqrt{m_{1} m_{2}}} \mathbf{p}_{1} \mathbf{p}_{2} .
$$

Next, setting the coordinate transformations

$$
\begin{aligned}
& \mathbf{x}_{i} \rightarrow\left(\frac{\hbar}{\omega_{i} m_{i}}\right)^{1 / 2} \mathbf{x}_{i} \\
& \mathbf{p}_{i} \rightarrow\left(\hbar \omega_{i} m_{i}\right)^{1 / 2} \mathbf{p}_{i}
\end{aligned}
$$


the Hamiltonian in Eq.(16 ) may be written in the form ( with $\omega=k \sqrt{\omega_{1} \omega_{2}}$ ),

$$
\mathbf{H}=\hbar\left(\frac{1}{2} \sum_{i}\left(\omega_{i} \mathbf{q}_{i}^{2}+\omega_{i} \mathbf{p}_{i}^{2}\right)+\omega \mathbf{p}_{1} \mathbf{p}_{2}\right) .
$$

The Hamilton's equations of motion results ( with $i=1,2 ; j \neq i$ )

$$
\begin{aligned}
\dot{\mathbf{x}}_{i} & =\omega_{i} \mathbf{p}_{j} \\
\dot{\mathbf{p}}_{i} & =-\omega_{i} \mathbf{x}_{i},
\end{aligned}
$$

leading to

$$
\ddot{x}_{i}=-\omega_{i} \mathbf{x}_{i}-\omega \omega_{j} \mathbf{x}_{j} .
$$

We may put the foregoing equation in the matrix form

$$
[\ddot{x}]=-[\omega][x],
$$

where

$$
\begin{aligned}
& {[x]=\left(\begin{array}{c}
\mathbf{x}_{1} \\
\mathbf{x}_{2}
\end{array}\right),} \\
& {[\ddot{x}]=\left(\begin{array}{c}
\ddot{\mathbf{x}}_{1} \\
\ddot{\mathbf{x}}_{2}
\end{array}\right),} \\
& {[\mathbf{x}]=\left(\begin{array}{c}
\mathbf{x}_{1} \\
\mathbf{x}_{2}
\end{array}\right),}
\end{aligned}
$$

and

$$
[\omega]=\left(\begin{array}{ll}
\omega_{1}^{2} & \omega \omega_{2} \\
\omega \omega_{1} & \omega_{2}^{2}
\end{array}\right) .
$$

Now, diagonalization of the Eq.(22) is obtained in the form

$$
[\ddot{X}]=-[\Omega][X],
$$

where $[X]=[A]^{-1}[x],[\Omega]$ is the diagonal matrix

$$
[\Omega]=\left(\begin{array}{cc}
\Omega_{1}^{2} & 0 \\
0 & \Omega_{2}^{2}
\end{array}\right)
$$

and $\Omega_{1}^{2}$ and $\Omega_{2}^{2}$ are the roots of the equation

$$
\left(\Omega^{2}-\omega_{1}^{2}\right)\left(\Omega^{2}-\omega_{2}^{2}\right)-\omega \omega_{1} \omega_{2}=0,
$$

namely,

$$
\begin{aligned}
& \Omega_{1}^{2}=\frac{\omega_{1}^{2}+\omega_{2}^{2}}{2}+\left[\frac{\omega_{1}^{2}-\omega_{2}^{2}}{2}+\omega \omega_{1} \omega_{2}\right]^{1 / 2}, \\
& \Omega_{2}^{2}=\frac{\omega_{1}^{2}+\omega_{2}^{2}}{2}-\left[\frac{\omega_{1}^{2}-\omega_{2}^{2}}{2}+\omega \omega_{1} \omega_{2}\right]^{1 / 2} .
\end{aligned}
$$

For $\Omega_{2}^{2}>0$, we must have $\omega<\omega_{1} \omega_{2}$ which is equivalent to $k^{2}<1$.

The matrix $[A]$ is gotten from

$$
[A]=\left(\begin{array}{cc}
1 & -\omega_{2} A \\
\omega_{1} A & 1
\end{array}\right),
$$

with $A$ given by

$$
A=-\frac{\omega_{1}^{2}-\omega_{2}^{2}}{2 \omega \omega_{1} \omega_{2}}+\left[\left(\frac{\omega_{1}^{2}-\omega_{2}^{2}}{2 \omega_{1} \omega_{2}}\right)^{2}+\frac{1}{\omega_{1} \omega_{2}}\right]^{1 / 2},
$$

and

$$
[A]^{-1}=\frac{1}{1+\omega_{1} \omega_{2} A^{2}}\left(\begin{array}{cc}
1 & \omega_{2} A \\
-\omega_{1} A & 1
\end{array}\right) .
$$

The substitution of Eqs. (28 -32) in the Eq. (27) yields the eigenvalue equation

$$
\ddot{X}_{i}=-\Omega_{i}^{2} X_{i} \quad, \quad i=1,2 .
$$

The application of these equations for the calculation of the variances

$$
\begin{aligned}
\Delta \mathbf{x}_{i} & =\sqrt{\left\langle\mathbf{x}_{i}^{2}>-\left\langle\mathbf{x}_{i}\right\rangle^{2}\right.}, \\
\Delta \mathbf{p}_{i} & =\sqrt{\left\langle\mathbf{p}_{i}^{2}>-<\mathbf{p}_{i}\right\rangle^{2}},
\end{aligned}
$$

allows us to obtain, for both oscillators initially in coherent states,

$$
\begin{gathered}
\left(\Delta \mathbf{x}_{1}(t)\right)^{2}=\frac{1}{2}\left(1+\omega_{1} \omega_{2} A^{2}\right)^{-2}\left[f_{11}^{2}+f_{12}^{2}+g_{11}^{2}+g_{12}^{2}\right], \\
\left(\Delta \mathbf{x}_{2}(t)\right)^{2}=\frac{1}{2}\left(1+\omega_{1} \omega_{2} A^{2}\right)^{-2}\left[f_{21}^{2}+f_{22}^{2}+g_{21}^{2}+g_{22}^{2}\right], \\
\left(\Delta \mathbf{p}_{1}(t)\right)^{2}=\frac{1}{2}\left(\omega_{1} \omega_{2}-\omega^{2}\right)^{-2}\left(1+\omega_{1} \omega_{2} A^{2}\right)^{-2}\left(\omega_{2} \dot{f}_{11}-\omega \dot{f}_{21}\right)^{2}+ \\
\left(\omega_{2} \dot{f}_{12}-\omega \dot{f}_{22}\right)^{2}+\left(\omega_{2} \dot{g}_{11}-\omega \dot{g}_{21}\right)^{2}+\left(\omega_{2} \dot{g}_{12}-\omega \dot{g}_{22}\right)^{2} \\
\left(\Delta \mathbf{p}_{2}(t)\right)^{2}=\frac{1}{2}\left(\omega_{1} \omega_{2}-\omega^{2}\right)^{-2}\left(1+\omega_{1} \omega_{2} A^{2}\right)^{-2}\left(\omega_{1} \dot{f}_{22}-\omega \dot{f}_{12}\right)^{2}+ \\
\left(\omega_{1} \dot{f}_{21}-\omega \dot{f}_{11}\right)^{2}+\left(\omega_{1} \dot{g}_{22}-\omega \dot{g}_{12}\right)^{2}+\left(\omega_{1} \dot{g}_{21}-\omega \dot{g}_{11}\right)^{2}
\end{gathered}
$$


where ( with $i, j=1,2, i \neq j)$

$$
\begin{aligned}
f_{i i} & =\cos \Omega_{i} t+\omega_{1} \omega_{2} A^{2} \cos \Omega_{j} t, \\
f_{i j} & =\left(\cos \Omega_{i} t-\cos \Omega_{2} t\right) \omega j A,
\end{aligned}
$$

and

$$
\begin{aligned}
& g_{i i}=h_{i i} \omega_{i}+h_{i j} \omega, \\
& g_{i j}=h_{i i} \omega+h_{i j} \omega_{j},
\end{aligned}
$$

with

$$
\begin{aligned}
& h_{i i}=\frac{\sin \Omega_{i} t}{\Omega i}+\omega_{1} \omega_{2} A^{2} \frac{\sin \Omega_{j} t}{\Omega j}, \\
& h_{i j}=\left(\frac{\sin \Omega_{i} t}{\Omega_{i}}-\frac{\sin \Omega_{j} t}{\Omega_{j}}\right) \omega_{j} A .
\end{aligned}
$$

Figs. (1-2) show the squeezing effect appearing in the variances $\Delta \mathbf{x}_{1}$ and $\Delta \mathbf{x}_{2}$ for a convenient set of parameters. Note that the effect is amplified in the variable $x_{1}$ when $k$ increases, as shown in Fig.(2). No squeezing is observed in the variable $p_{1}$, for same values of parameters used in Fig.(2), as shown in Fig.(3) - the same occurring for variable $p_{2}$, (not shown in figures).

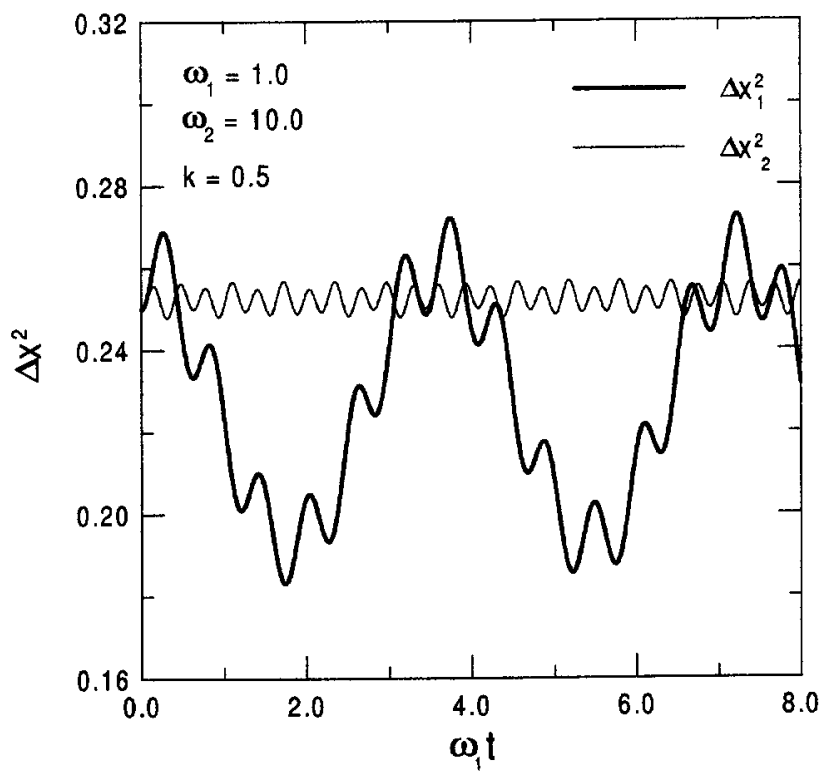

Figure 1. Plots of variances $\Delta \mathbf{X}_{1}^{2}$ (thin line) and $\Delta \mathbf{X}_{2}^{2}$ (thick line), for a set of parameters $\left(k=0,5 ; w_{1}=\right.$ $\left.1.0 ; w_{2}=10.0\right)$, showing squeezing effect in the variable $x_{1}$.

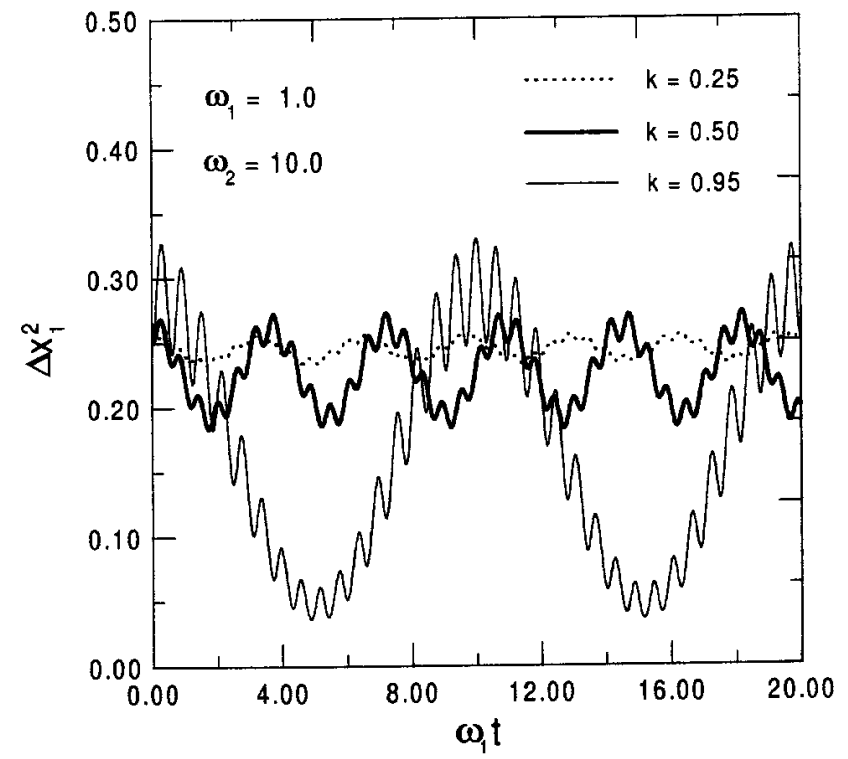

Figure 2. Same as Fig.1 for variance $\Delta \mathbf{X}_{1}^{2}$, for various values of $k$, showing the amplification of the effect when $k$ increases.

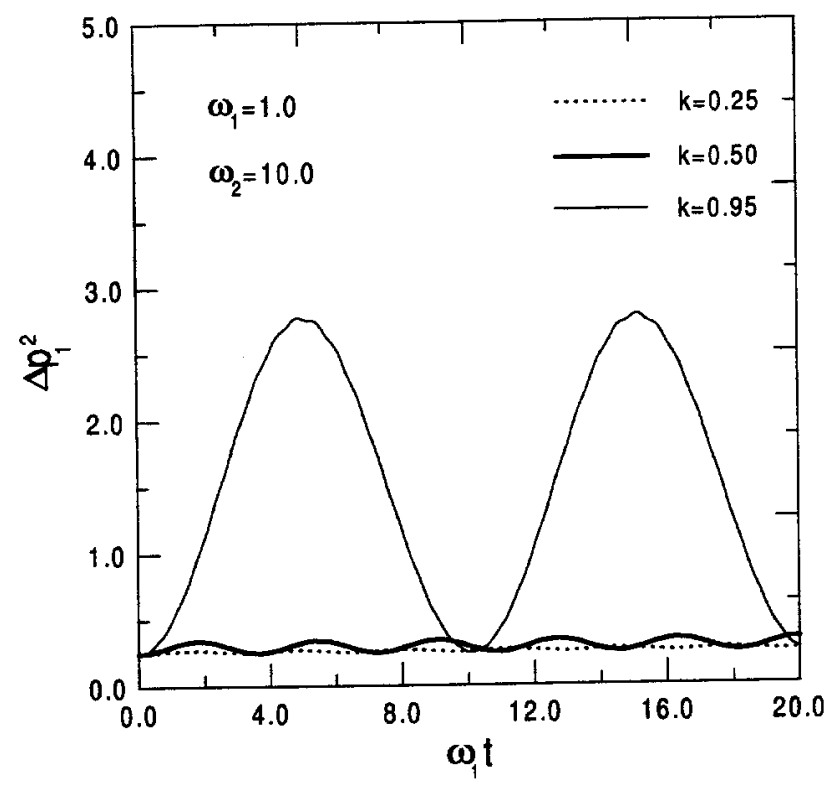

Figure 3. Same as Fig. 2 for variable $p_{1}$, showing the absence of the effect in this observable.

\section{Comments and Conclusion}

We have studied the squeezing effect appearing in two coupled oscillators as shown in Figs.(1,2). When, e.g., the effect is generated in the first oscillator [see Fig.(1)] it is transferred to the second oscillator if $\omega_{1}>\omega_{2}$ ( not shown in figures ). The present scheme explicitly involves neither nonlinearities nor time-dependent parameters, whose presences cause squeezing $[1,5,8]$. So, a raising question is: what is the origin of squeezing in our system? To answer this point, one should 
remember that: (i) squeezing is essentially a nonlinear effect [1], hence some nonlinearity might be hidden somewhere in our coupled oscillators, since it is not explicitly present in the Hamiltonian given in Eq.(19); (ii) the effect also appears in "similar" systems, which results when our oscillator-oscillator system is replaced by an oscillator-atom (or field-atom) system [14]. In fact, it can be shown that in our case, as in [14], nonlinearities are hidden in the whole system and, when solving the Heisenberg equations of motion for the fieldoperators, $\mathbf{a}$ and $\mathbf{a}^{+}$, we obtain from the Heisenberg equations of motion a set of coupled differential equations whose iterative solutions will show nonlinearities (e.g., quadratic terms in $\mathbf{a}$ and $\mathbf{a}^{+}$). The same occurs for the field-atom system when one employs the JaynesCummings model Hamiltonian [14], being also neither nonlinear nor time-dependent. In both cases (our coupled oscillators and the coupled field-atom system of Ref. [14]), the squeezing effect originates from nonlinear couplings in these systems. In the present case, squeezing may be transferred back and forth (Fig.2). We will show elsewhere that, if the system involves a time-dependent parameter, the effect may be transferred permanently, with no returning.

\section{Acknowledgments}

This paper was partially supported by the Conselho Nacional de Desenvolvimento Científico e Tecnológico (CNPq), PRONEX/CNPq.

\section{References}

[1] H.N. Hollenhorst, Phys. Rev. D19, 1669 (1979); D. Stoler, Phys. Rev. D1, 3217 (1970); E. Y. C. Lu, Lett. novo Cimento 2, 1241 (1971); H. P. Yuen, Phys. Rev. A13, 2226 (1976); D. F. Walls, Nature 306, 141 (1983); R. Loudon and P. L. Knight, J Mod. Opt. 34, 709 (1987); A.F.R. de Toledo Piza, Phys. Rev. A51, 1612 (1995).

[2] R. L. Robinson, Science 230, 927 (1985), 233, 280 (1986); R. E. Sluscher, L. W. Hollberger, B. Yurke, J. C. Mertz and F. Valley, Phys. Rev. Lett. 55, 2409 (1986); R. M. Shelby, M. D. Levenson, AS. M. Perlmutter, R. G. De Voe and D. F. Walls, Phys. Rev. Lett. 57, 691 (1986).

[3] R. J. Glauber and M. Levenstein, Phys. Rev. A43, 467 (1991);
[4] R.J. Klauder and B. O. Skagerstan, Eds., "Coherent States: Application in Physics and Mathematical Physics "(World Scient., Singapore, 1985), p.16; R. J. Glauber, "Quantum Theory of Particle Trapping by Oscillating Fields" - Lyman Labotory of Physics, Harvard Univ. 021381/HUTP - 91/B001 (1991).

[5] G. T. Moore, J., Math Phys. 11, 2679 (1970); A. K. Rajagopal and J. T. Marshall, Phys. Rev. A26, 2977 (1982); G. A. D. Janussis and B. S. Bartzis, Phys. Rev. Lett. A12, 263 (1988); V. V. Dodonov, A. B. Klimov and V. I. Manko, Phys. Lett. A149, 225 (1990); Phys. Lett. A244, 517 (1998); Phys. Scripta 58, 469 (1998); J. Phys. A: Math. Gen. 31, 9835 (1998); H. Takahashi, Adv. Commun. Syst. 1, 227 (1965); J. Janszky and P. Adam, Phys. Rev. A46, 6091 (1992); Phys. Lett. A174, 368 (1993); C. F. Lo, Phys. Lett. A162, 299 (1992); Phys. Rev. A47, 733 (1993); M. Havukainen and S. Stenholm, Phys. Lett. A60, 621(1999).

[6] B. Baseia, Phys. Lett. A170, 311 (1992); Phys. Rev. A10, 4097 (1989); B. Baseia, S. S. Mizrhai and M. H. Y. Moussa, Phys. Rev. A46, 5885 (1992); Intern. J. Mod. Phys. 8, 1563 (1994); A. L. Brito and B. Baseia, Quantum Opt. 4, 201 (1992 ); B. Baseia, R. Vyas and Bagnato, Quantum Opt. 5, 155 (1993); Physica A197 364 (1993); B. Baseia, C.M. Dantas, R. Vyas and V. S. Bagnato, Quantum Opt. 6, 73 (1994); C.M. Dantas, I. Pedrosa and B. Baseia, Phys Rev. A 45, 1320(1992).

[7] A. L. Silva, B. Baseia and G. C. Marques, Mod Phys. Lett. B9, 1213 (1995).

[8] A. R. Gomes, B. Baseia and G. C. Marques, Mod Phys. Lett. B9, 999 (1995).

[9] C. F. Lo and R. Sollie, Phys. Lett. A194, 7 (1994).

[10] A. K. Ekert and P. L. Knight, Am. J. Phys. 57, 692 (1989).

[11] E. T. Jaynes and Cummings, Proc. IEEE, 51, 63 (1963).

[12] See, e. g., H. M. Nussenzveig, "Introduction to Quantum Optics" (Gordon and Breach, NY,1973), Chap.3.

[13] P. W. Milloni, J. R. Ackerhalt and H. W. Galbraith, Phys. Rev. Lett. 50, 966 (1983); R. F. Fox, J. C. Eidson, Phys. Rev. A36, 4321 (1987); R. Vyas and S. Singh, Phys. Rev. A33, 375 (1986); S. J. D. Phoenix, J Mod. Opt. 36, 1163 (1989); J. S. Peng, Phys. Rev. A45, 3289 (1992); K. Zaheer and M. S. Zubairy, Opt. Comm. 73, 325 (1988); 78, 346 (1990).

[14] S. M. Barnett and P. L. Knight, Phys. Scripta, T12, 5 (1988). 